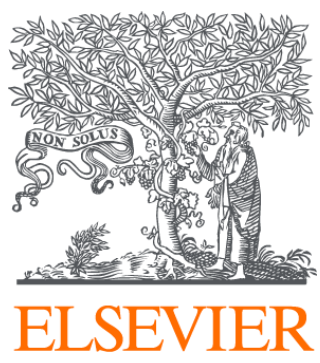

Since January 2020 Elsevier has created a COVID-19 resource centre with free information in English and Mandarin on the novel coronavirus COVID-

19. The COVID-19 resource centre is hosted on Elsevier Connect, the company's public news and information website.

Elsevier hereby grants permission to make all its COVID-19-related research that is available on the COVID-19 resource centre - including this research content - immediately available in PubMed Central and other publicly funded repositories, such as the WHO COVID database with rights for unrestricted research re-use and analyses in any form or by any means with acknowledgement of the original source. These permissions are granted for free by Elsevier for as long as the COVID-19 resource centre remains active. 


\title{
Deficient synthesis of melatonin in COVID-19 can impair the resistance of coronavirus patients to mucormycosis
}

\author{
Amarnath Sen \\ 40 Jadunath Sarbovouma Lane, Kolkata 700035, India
}

\section{A R T I C L E I N F O}

\section{Keywords:}

Coronavirus

COVID-19

Mucormycosis

Melatonin

Tryptophan

'Black fungus'

\begin{abstract}
A B S T R A C T
In addition to uncontrolled diabetes and the excess use of corticosteroids, it is believed that other factors may be responsible for the recent spurt of COVID-19 associated mucormycosis (CAM). In the present paper it is argued that COVID-19 increases the susceptibility of the patients to mucormycosis by augmenting the virulence factors of the mucor species, where deficient synthesis of melatonin plays a key role. Melatonin is synthesized from tryptophan via the serotonin pathway and melatonin deficiency in COVID-19 arises from the faulty absorption of tryptophan from the food because SARS-CoV-2 downregulates angiotensin-converting enzyme-2, the chaperone of the transporter of tryptophan. The enhanced fungal virulence in COVID-19 can be mitigated by correcting the melatonin deficiency as melatonin can prevent iron acquisition of the mucor species and inhibit their morphological transition from the yeast to the virulent hyphal form, given the fact that melatonin is an iron chelator, calmodulin blocker and inhibitor of myeloperoxidase as well as inhibitor of ferroptosis and pyroptosis. Also, by lowering the expression of glucose-regulated protein 78 and by inhibiting the suppression of T-cell immunity, melatonin can further increase the resistance of the patients to mucormycosis. Accordingly, clinical trials should be carried out on tryptophan supplementation, administration of selective serotonin reuptake inhibitors (to increase serotonin, the precursor of melatonin), and exogenous melatonin to find out how they perform in eliminating or reducing the propensity of the coronavirus patients to CAM.
\end{abstract}

\section{Introduction}

Mucormycosis (earlier called Zygomycosis) is a potentially fatal opportunistic infection caused by the fungi of the order Mucorales, which are commonly found in the soil and decomposing organic matters. The major risk factors of mucormycosis include uncontrolled diabetes mellitus (ketoacidosis and, in general, acidosis of any aetiology), immunosuppression, trauma and burn, use of corticosteroids, neutropenia, organ transplantation, multiple blood transfusion, chemotherapy and deferoxamine therapy in hemodialysis [1-3]. The higher incidence of mucormycosis in India is probably because of the fact that around one in six people with diabetes in the world is from India and around $82 \%$ of mucormycosis patients are diabetic [4-6].

Incidentally, a spike in COVID-19 associated mucormycosis (CAM) has recently been observed in India and approximately $71 \%$ of the global cases of CAM are from India [7]. The higher incidence of CAM in India has been primarily attributed to uncontrolled diabetes and the suppression of immunity from the excessive use of steroids [5]. Other COVID-19 associated fungal infections like aspergillosis and candidiasis have also been reported, though in relatively small numbers [8-9].

Interestingly, mucor species are dimorphic and exhibit either yeast (unicellular) or virulent hyphal (multicellular) form depending upon the conditions. Normally, aerobic condition favours the growth in the hyphal form [10]. In the invasive hyphal form, the hyphae are attached to the endothelium through coupling of glucose-regulated protein (GRP78, an endothelial cell receptor) on the host and spore coat homolog (CotH) protein on the fungus and eventually, the disease may manifest as rhinoorbital, rhino-orbital-cerebral, pulmonary or disseminated mucormycosis $[7,11,12]$. Mucormycosis infection is characterized by extensive angioinvasion leading to vessel thrombosis and tissue necrosis and is often presented with black necrotic lesions (that is why mucormycosis is popularly known as 'black fungus'), and the spread and severity of the infection are controlled by the virulence factors of the fungi, the factors which increase their virulence in the host. One of the major virulence factors $[1,3,11,13]$ of the mucor species is the iron acquisition system, the system of 'stealing' iron from the host, given the fact that iron is an essential element for the survival of most of the microbes. Indeed, it has been found that fungi undergo apoptosis in iron-deprived conditions

E-mail address: amarns2@yahoo.co.in. 
$[3,13]$. Accordingly, the strategy of limiting iron availability to the mucor species is an important host defense mechanism (nutritional immunity), and serum and other biological fluids are normally fungistatic but iron addition abolishes this benefit [14]. In humans the predominant forms of iron present are sequestered/complexed with proteins such as transferrin in the circulating fluid (extracellular iron) and heme and ferritin in the cells (intracellular iron). A small amount of free, redox-active and ligand-exchangeable iron (labile iron pool) also exists as a transitory pool to regulate iron metabolism and homeostasis, though any excess free iron is rapidly sequestered [1,3,11].
Interestingly, fungi have developed three mechanisms [1,3,13-15] of iron acquisition from the host for their survival: a) reductive iron uptake, b) siderophore mediated uptake and c) uptake from heme by hemeoxygenase (by degrading heme) or by using hemophores. Reductive iron uptake from sequestered ferric iron has three steps - reduction of ferric iron to soluble ferrous form by ferric reductase, re-oxidation to the ferric form by ferroxidase and transport into the cell by permease. On the other hand, siderophores/hemophores are low molecular weight proteins which can chelate iron by grabbing it from the protein-bound iron of the host as the iron affinity of some siderophores/hemophores can be higher

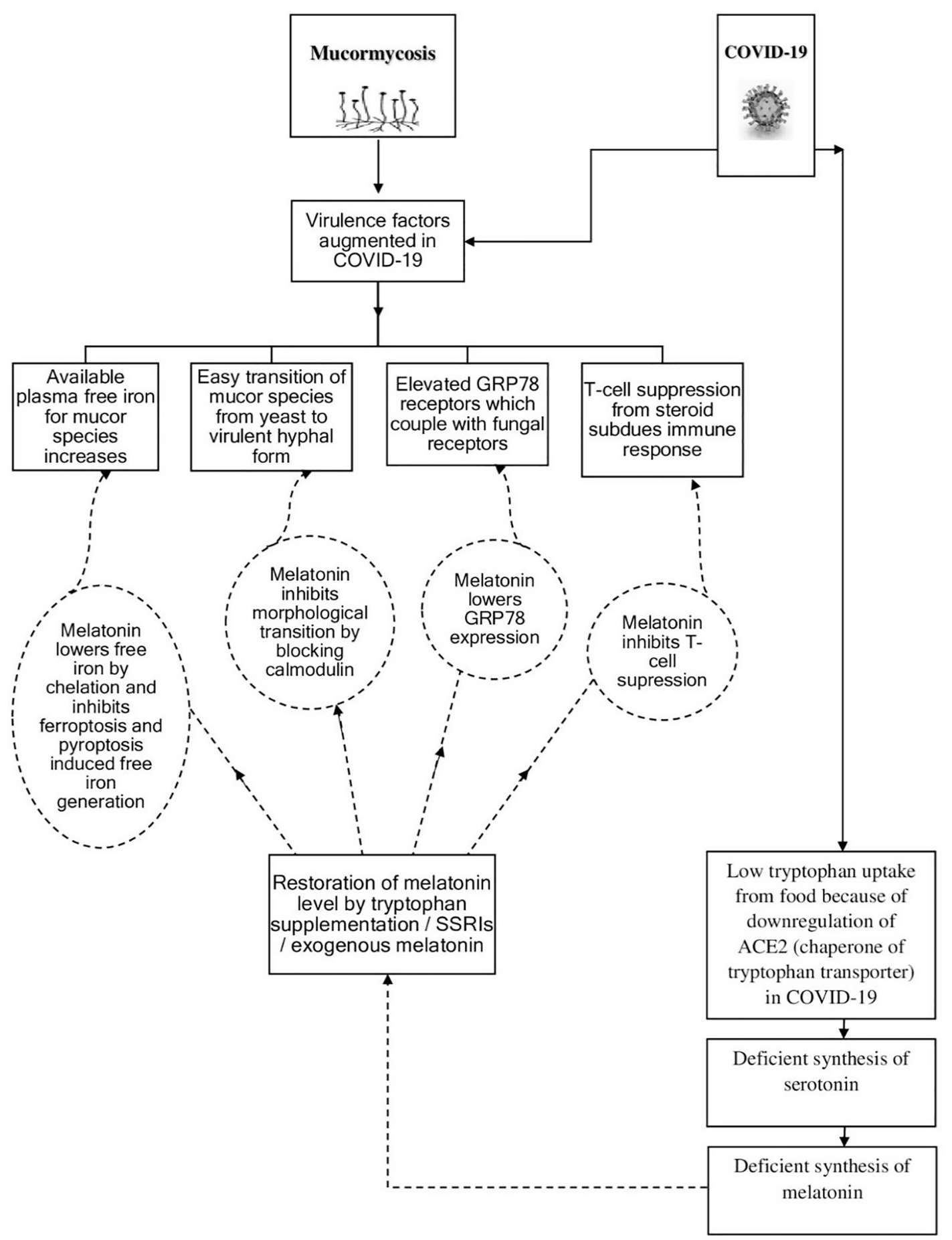

Fig. 1. Schematic outline of the hypothesis depicting how COVID-19 makes the patients susceptible to CAM and the restoration of melatonin deficiency in coronavirus patients protects them from the deadly menace [CAM $\rightarrow$ COVID-19 Associated Mucormycosis, GRP $78 \rightarrow$ Glucose-Regulated Protein 78 (78 kDa molecular weight), ACE2 $\rightarrow$ Angiotensin-Converting Enzyme 2, SSRI $\rightarrow$ Selective Serotonin Reuptake Inhibitor, T-cell $\rightarrow$ Thymus-derived lymphocyte]. 
than the sequestration affinity of iron in the host.

\section{Hypothesis}

Given the fact that diabetes is a perennial problem in India and the long-term use of steroid is not uncommon as a large number of people suffer from arthritis and other chronic inflammatory diseases, it has been suggested by some researchers that in addition to uncontrolled diabetes and excess use of steroid, other factors like the use of zinc supplements, contaminated water in humidifiers, impure oxygen etc. may also be responsible for the spike in CAM [5], though the jury is still out.

In the present paper, it is proposed that other than uncontrolled diabetes and compromised immunity from steroid use, COVID-19 per se augments the virulence of the mucor species, where melatonin deficiency (from reduced synthesis in coronavirus patients) plays a major role. It is further argued that by correcting the melatonin deficiency, the fungal virulence can be contained and, consequently, the susceptibility of the patients to CAM can be eliminated or reduced significantly.

\section{How does melatonin synthesis dip in COVID-19?}

The formation of melatonin begins with the absorption of tryptophan (an essential amino acid) from the food. After absorption, tryptophan is first converted to 5-hydroxytryptophan (enzyme: tryptophan hydroxylase), then to serotonin (enzyme: decarboxylase) followed by $\mathrm{N}$-acetylserotonin (enzyme: aralkylamine $\mathrm{N}$-acetyltransferase, AANAT) and finally to melatonin (enzyme: acetylserotonin O-methyltransferase, ASMT) [16]. Two enzymes (AANAT and ASMT), found primarily in the pineal gland, transform serotonin to melatonin (mostly in the darkness) and the synthesized melatonin is released from the pineal gland into the bloodstream mainly during nighttime. Incidentally, many other extrapineal sites like cerebellum, retina, skin, gastrointestinal tract, immune cells, thymus, thyroid, bone marrow, liver, spleen, kidney, lungs, pancreas, heart and airway epithelia also generate melatonin, some even in larger quantities than the pineal gland, though most of the extrapineal melatonin is not integrated into the circulation $[17,18]$.

Interestingly, in the synthesis of melatonin, the very first step of absorption of tryptophan from the food is not so straightforward. The uptake of tryptophan needs a transporter $\mathrm{B}^{0} \mathrm{AT} 1$ (Broad neutral (0) Amino acid Transporter 1), where ACE2 (Angiotensin-Converting Enzyme 2) performs a key role as a chaperone (a protein that aids in proper folding of another protein) for $\mathrm{B}^{0} \mathrm{AT} 1$ [19]. As SARS-CoV-2 is known to downregulate ACE2 by docking on it, the absorption of tryptophan should be significantly reduced in coronavirus patients (Fig. 1). Hence, tryptophan deficiency in COVID-19 is expected to give rise to serotonin deficiency [20], and consequently, to melatonin deficiency.

\section{How is the iron acquisition system of mucor species modified in COVID-19?}

Iron acquisition system is one of the important virulence factors of the mucor species. As iron is an essential material for the fungi, there is a competition between the pathogens and the host for the same, and as discussed earlier, the host iron is almost completely sequestered and a little free iron (labile iron pool) is available for the pathogens, which is normally insufficient for their growth and virulence. However, if the disease per se increases the free iron level in blood, it becomes easier for the pathogens to gain entry into the host and grow. Indeed, COVID-19 creates such favourable conditions for the growth and invasion of the mucor species.

Though ferritin is known to store iron intracellularly, a small amount of ferritin is also available in the serum of healthy subjects, which increases significantly (hyperferritinemia) in inflammation of any etiology as an acute phase reactant [21]. The serum ferritin is of light-chain variety (molecular weight $19 \mathrm{kDa}$, in contrast to the intracellular heavy-chain variety of molecular weight $21 \mathrm{kDa}$ ) and during inflammation, serum ferritin may be produced from hepatocytes, Kupffer cells, proximal tubular renal cells and macrophages. Also, exaggerated synthesis or secretion of ferritin has been reported to occur from various stimuli like pro-inflammatory cytokines, prostaglandins, reactive oxygen species (ROS), oxidants, growth factors and hypoxia [21]. As COVID-19 is a hyperinflammatory disease, high serum ferritin is quite common in coronavirus patients [22]. In such inflammatory states, mainly three sources take part in elevating the plasma free iron (labile plasma iron, LPI). First, during inflammation when low molecular weight serum ferritin is formed from high molecular weight intracellular ferritin, the latter releases a substantial portion of its inner core iron, which increases the free iron level in the blood [21,22]. Second, inflammation reduces RBC half-life, which leads to macrophagemediated RBC phagocytosis and eventually, an increase in the free iron in the plasma $[23,24]$. Third, free iron is also generated from myeloperoxidase (MPO) heme degradation [23], given the fact that $\mathrm{MPO}$, an enzyme primarily released by neutrophils to protect against pathogens, is increased during inflammation. Interestingly, the excess free iron thus produced can assist in further raising the free iron by generating highly reactive hydroxyl radical $\left(\mathrm{HO}^{\bullet}\right)$ via Fenton reaction from $\mathrm{H}_{2} \mathrm{O}_{2}$ and $\mathrm{Fe}^{2+}$ and/or via Haber-Weiss reaction from $\mathrm{H}_{2} \mathrm{O}_{2}$ and superoxide anion $\left(\mathrm{O}_{2}{ }^{\bullet}\right)$ in presence of $\mathrm{Fe}^{2+} / \mathrm{Fe}^{3+}$, resulting in the $\mathrm{HO}^{\circ}$ induced damage of cellular proteins, lipids (lipid peroxidation) and nucleic acids $[24,25]$. The process can lead to ferroptosis, a type of inflammatory programmed cell death with accumulation of lipid peroxides arising from degradation of lipids in presence of free iron and ROS. Ferroptosis in COVID-19 results in aggravation of the inflammation $[26,27]$ and, in effect, further generation of inflammation induced free iron in the plasma. Hence, in coronavirus patients the labile plasma iron increases by various means and the mucor species take advantage of this easily available free iron for their growth and virulence.

\section{How can fixing melatonin deficiency restrict iron uptake by the mucor species?}

Melatonin, a multifunctional neuroendocrine hormone, is celebrated mainly for its anti-inflammatory (with some useful pro-inflammatory characteristics), antioxidant, anti-aging, analgesic, free radical scavenging and oncostatic effects $[17,18]$. Melatonin modifies circadian rhythm, mood, sleep, appetite, reproduction, immune responses, cardiac functions etc. in receptor independent/receptor dependent pathways $[17,18,28]$. In the present context, melatonin has multiple roles in containing the plasma free iron. First, melatonin helps in reducing plasma free iron in SARS-CoV-2 patients by chelating excess iron (both $\mathrm{Fe}^{2+}$ and $\mathrm{Fe}^{3+}$ ) as melatonin is an effective metal chelator $[29,30]$. Second, melatonin, being a potent anti-inflammatory agent, can help in shrinking the labile iron pool by i) limiting free iron generation from myeloperoxidase (MPO) heme destruction during inflammation by blocking the enzyme MPO [23] ii) restricting free iron production from inflammation induced RBC degradation $[23,24]$ and iii) lowering the formation of serum ferritin $[31,32]$ (an acute phase reactant of inflammatory processes), which is involved in elevating plasma free iron. Melatonin ameliorates inflammation by blocking pro-inflammatory cytokines such as interleukin IL-1 $\beta$, IL-2, IL-6, tumor necrosis factor- $\alpha$ $(\mathrm{TNF}-\alpha)$ and interferon- $\gamma($ IFN- $\gamma$ ) $[16,17,28,33]$. Melatonin also restrains inflammation by inhibiting prostaglandin synthesis, production of adhesion molecules, generation of pro-inflammatory cytokines from lipopolysaccharides (LPS) and by downregulating cyclooxygenase and NLRP3 inflammasome [16,17,28,33]. Inflammasomes, which are intracellular multiprotein complexes, act as pattern-recognition receptors (PRRs) to sense pathogens and are triggered by various stimuli like pathogens, endogenous cytokines and damaged cells [33,34]. NLRP3 inflammasome mediated activation of nuclear transcription factor-kappa B (NF-kB) leads to pyroptosis, a type of programmed cell death arising from infection induced activation of inflammasomes and 
release of pro-inflammatory cytokines [33,34]. Incidentally, in COVID19 , pyroptosis gives rise to exaggerated inflammation and melatonin can block pyroptosis by antagonizing NF- $\mathrm{kB}$ and NLRP3 signaling $[33,34]$. In addition to lowering proinflammatory cytokines and scavenging ROS and $\mathrm{H}_{2} \mathrm{O}_{2}$ [29], melatonin, being an inhibitor [35] of ferroptosis (programmed cell death in presence of lipid peroxides, free iron and ROS), can lessen ferroptosis induced inflammation in coronavirus patients [27]. Hence, melatonin by chelating excess free iron and by ameliorating inflammation in multiple ways can reduce labile plasma iron (Fig. 1) and can force the mucor species to starve and, as expected, melatonin deficiency in COVID-19 deprives the coronavirus patients of the aforesaid benefits and makes them susceptible to mucormycosis.

In this context, it may be noted that zinc is also an essential nutrient for the fungi as zinc is the cofactor of several enzymes. However, in CAM, any role of excess zinc in the virulence of the mucor species may possibly be ruled out as melatonin deficiency per se leads to the lowering of zinc level in blood [36] and indeed, a significant proportion of coronavirus patients is zinc deficient and zinc deficiency leads to increased severity of the disease [37].

\section{How can restoration of melatonin reduce the likelihood of yeast to hyphal transition of the fungi?}

Another critical factor in the virulence of mucor species is the switching of their mode of growth from the yeast (unicellular) to the virulent filamentous hyphal (multicellular) form $[3,13]$. Calcineurin, an important fungal regulator of calcium homeostasis and signaling, is responsible for the transition of the mucor species from the yeast to the tissue invasive hyphal form $[10,13,38]$. Calcineurin is a $\mathrm{Ca}^{2+}$ and calmodulin-dependent serine/threonine protein phosphatase, where calmodulin (calcium-modulated protein) is a sensor protein that transduces calcium signals into appropriate outputs through calmodulinbinding proteins (like calcineurin), calmodulin-dependent protein kinases, and histone deacetylases [10,38]. When calcineurin function is inhibited, growth shifts to the less virulent yeast form [10,38]. Any material that blocks calmodulin is also expected to show antifungal activity $[38,39]$. Incidentally, melatonin is a calmodulin inhibitor [39] and hence, can diminish the fungal virulence by restraining the morphological transition of mucor species from the yeast to the virulent hyphal form (Fig. 1).

\section{How can the victims of diabetes and patients receiving steroids benefit from melatonin?}

It is known that acidic environment helps in the growth of the mucor species as acidosis temporarily disrupts the capacity of transferrin to bind iron owing to proton mediated dissociation of iron from transferrin $[12,40]$ resulting in the generation of free iron. Hence, in uncontrolled diabetes, ketoacidosis generates excess free iron and produces the right environment for the proliferation of mucor species, where melatonin has a prominent role in chelating the free iron and making it unavailable to the mucor species.

Also, the expressions of GRP 78 (endothelial cell receptor on the host) and CotH (receptor on the fungal surface) are increased in the presence of high glucose and iron, further increasing the propensity of diabetic patients to mucormycosis $[12,41]$. Melatonin provides an added advantage to the diabetic patients by lowering the GRP78 expression [41] and, hence, attenuating the host-fungus coupling (Fig. 1). Melatonin also lowers pyroptosis induced endothelial cell damage [33] and hence, restricts easy invasion of the mucor species through the damaged regions of the endothelial cells. Incidentally, the restoration of melatonin level should be more helpful to the diabetic patients as they have lower blood melatonin than healthy people [42].

Glucocorticoid like dexamethasone is known to suppress immunity mainly by inhibiting IL-2 mediated T-cell proliferation. Melatonin has been found to enhance T-cell associated immune responses and reduce dexamethasone-induced immunosuppression [43]. It has been found that T-cells have a major role in developing immunity against fungi [44] and hence, the restoration of melatonin level in coronavirus patients is expected to lessen the steroid induced immunosuppression as well as increase the resistance of the patients to mucormycosis (Fig. 1).

\section{How to correct melatonin deficiency in SARS-CoV-2 patients}

The preferred way to treat the melatonin deficiency is to restore the tryptophan level to normal by supplementation so that the serotonin level and consequently, the melatonin level go up [20]. In case of severe COVID-19, when there is hardly any absorption of tryptophan, parenteral supplementation of tryptophan may be considered. It should be noted that in SARS-CoV-2 patients, selective serotonin reuptake inhibitors (SSRIs) may not significantly raise the level of serotonin, the precursor of melatonin, as SSRIs do not act well when there is tryptophan deficiency in the system [20]. On the other hand, exogenous melatonin should help in correcting the melatonin deficiency, though the serotonin deficiency remains unchanged with other possible consequences [20]. As exogenous melatonin has a short half-life (1-2 h), different formulations like extended-release and combined immediate and extended-release may be better options for fixing the melatonin deficiency [45]. It may be noted that exogenous melatonin is safe and some mild side effects like daytime sleepiness, headache, nausea and drowsiness have been reported in case of higher doses and extendedrelease formulations [45]. Hence, clinical trials (prophylactic as well as therapeutic) of tryptophan supplementation, SSRIs (excluding any combination of tryptophan and SSRI to guard against serotonin syndrome [20]) and exogenous melatonin should be conducted on coronavirus patients to find out how they perform in saving the patients from the deadly consequences of mucormycosis.

In this context, it is worthy to note that exogenous melatonin has been touted by many as the silver bullet for COVID-19 and using network medicine methodologies along with clinical and multi-omics (genomics, proteomics etc.) observations, it has been suggested that supplementation with melatonin is associated with $28 \%$ reduced likelihood (52\% for black Americans) of being infected with SARS-CoV-2 [46]. Melatonin may also attenuate SARS-CoV-2 replication by modifying the growth factor receptor signaling essential for viral proliferation [47]. Given the high potential of melatonin in reducing the severity of COVID-19, at least nine clinical trials (therapeutic and prophylactic) of exogenous melatonin on coronavirus patients are under progress [48]. Hence, melatonin is expected to play a pivotal role not only in reducing the severity of COVID-19, but also in lessening or eliminating the susceptibility of the coronavirus patients to mucormycosis.

\section{Conclusion}

It is suggested that COVID-19 enhances the virulence of mucor species and increases the susceptibility of the patients to mucormycosis, where melatonin deficiency is a key factor. The deficiency of melatonin in COVID-19 arises from impaired absorption of tryptophan (the precursor of serotonin and melatonin) from the food as ACE2, the chaperone of tryptophan transporter $\mathrm{B}^{0} \mathrm{AT} 1$, is downregulated in coronavirus patients. The melatonin deficiency in SARS-CoV-2 patients provides a significant advantage to the mucor species in their growth and virulence owing to the creation of a milieu favourable for their smooth access to iron, an essential nutrient, as well as for their facile transition from the yeast to the virulent hyphal form. The iron (labile plasma iron) acquisition of the mucor species can be restricted by correcting the melatonin deficiency, as melatonin is a good iron chelator and a potent antiinflammatory agent, which restricts inflammation induced generation of free plasma iron. Furthermore, the morphological transition of the mucor species from the yeast to the invasive hyphal form is inhibited by melatonin, which blocks calmodulin, an important sensor protein involved in the transition. In addition, melatonin can further help the 
diabetic patients by lowering the expression of GRP 78 receptors (increased in diabetic patients), which are exploited by the mucor species to invade the host cells. Also, the T-cell suppression owing to steroid use is blunted by melatonin. Hence, with a view to fixing the melatonin deficiency and consequently, eliminating or reducing the susceptibility of the patients to CAM, clinical trials (both prophylactic and therapeutic) of tryptophan supplementation, administration of SSRIs, and exogenous melatonin should be conducted on SARS-CoV-2 patients to find out the best regimen effective against CAM.

\section{Source of funding}

Nil

\section{Consent statement/Ethical approval}

Not required

\section{Declaration of Competing Interest}

The author declares that he has no known competing financial interests or personal relationships that could have appeared to influence the work reported in this paper.

\section{References}

[1] Ibrahim A, Spellberg B, Edwards Jr J. Iron acquisition: a novel prospective on mucormycosis pathogenesis and treatment. Curr Opin Infect Dis. 2008;21(6): $620-5$.

[2] Revannavar SM, P S S, Samaga L, V K V. COVID-19 triggering mucormycosis in a susceptible patient: a new phenomenon in the developing world? BMJ Case Rep 2021;14(4):e241663. https://doi.org/10.1136/bcr-2021-241663.

[3] Ibrahim AS, Spellberg B, Walsh TJ, Kontoyiannis DP. Pathogenesis of mucormycosis. Clin Infect Dis 2012;54(S1):S16-22.

[4] Skiada A, Pavleas I, Drogari-Apiranthitou M. Epidemiology and diagnosis of mucormycosis: an update. J Fungi 2020;6(4):265. https://doi.org/10.3390/ jof6040265.

[5] Deekshith N P, Sharma S, Dixit M, Kambli K, Deshpande A, Balsubramanian S. SciSimplified: amid growing threat of mucormycosis, debates rise over potential causes and names. 2021; 25 May. https://weather.com/en-IN/india/health/ news/2021-05-26-growing-threat-of-mucormycosis.

[6] Jain N. Over 21\% black fungus patients non-Covid. 2021; 1 June. https:// www. tribuneindia.com/news/ludhiana/over-21-black-fungus-patients-non-covid261961.

[7] John TM, Jacob CN, Kontoyiannis DP. When uncontrolled diabetes mellitus and severe COVID-19 converge: the perfect storm for mucormycosis. J Fungi 2021;7(4): 298. https://doi.org/10.3390/jof7040298.

[8] Arastehfar A, Carvalho A, van de Veerdonk FL, Jenks JD, Koehler P, Krause R, et al COVID-19 associated pulmonary aspergillosis (CAPA) - from immunology to treatment. J. Fungi 2020;6(2):91. https://doi.org/10.3390/jof6020091.

[9] Arastehfar A, Carvalho A, Nguyen MH, Hedayati MT, Netea MG, Perlin DS, et al. COVID-19-associated candidiasis (CAC): an underestimated complication in the absence of immunological predispositions? J. Fungi 2020;6(4):211. https://doi. org/10.3390/jof6040211.

[10] Lee S C, Li A, Calo S, Heitman J. Calcineurin plays key roles in the dimorphic transition and virulence of the human pathogenic zygomycete mucor circinelloides. PLoS Pathog. 2013; 9(9): e1003625.

[11] Almeida RS, Wilson D, Hube B. Candida albicans iron acquisition within the host. Yeast research. 2009;9(7):1000-101.

[12] Singh AK, Singh R, Joshi SR, Misra A. Mucormycosis in COVID-19: a systematic review of cases reported worldwide and in India. Diabetes \& Metabolic Syndrome: Clinical Research \& Reviews. 2021;15(4):102146. https://doi.org/10.1016/j dsx.2021.05.019.

[13] Petrikkos G, Tsioutis C. Recent advances in the pathogenesis of mucormycoses. Clin Ther 2018;40(6):894-902

[14] Symeonidis AS. The role of iron and iron chelators in zygomycosis. Clin Microbio Infect 2009;15(5):26-32.

[15] Stanford FA, Voigt K. Iron assimilation during emerging infections caused by opportunistic fungi with emphasis on mucorales and the development of antifungal resistance. Genes 2020;11(11):1296. https://doi.org/10.3390/genes11111296.

[16] do Amaral FG, Cipolla-Neto J. A brief review about melatonin, a pineal hormone. Arch Endocrinol Metab. 2018;62(4):472-9.

[17] Tordjman S, Chokron S, Delorme R, Charrier A, Bellissant E, Jaafari N, et al Melatonin: pharmacology, functions and therapeutic benefits. Curr Neuropharmacol 2017;15(3):434-43.

[18] Hardeland R. Melatonin - more than just a pineal hormone. Biomed J Sci \& Tech Res. 2017;1(4):994-7.
[19] Bröer S. The role of the neutral amino acid transporter B AT1 (SLC6A19) in Hartnup disorder and protein nutrition. IUBMB Life 2009;61(6):591-9.

[20] Sen A. Does serotonin deficiency lead to anosmia, ageusia, dysfunctional chemesthesis and increased severity of illness in COVID-19? Med. Hypotheses. 2021;153:110627. https://doi.org/10.1016/j.mehy.2021.110627.

[21] Kernan K F, Carcillo J A. Hyperferritinemia and inflammation. International Immunology, 2017(9): 401- 409.

[22] Ruscitti P, Berardicurti O, Di Benedetto P, Cipriani P, Iagnocco A, Shoenfeld Y, et al. Severe COVID-19, another piece in the puzzle of the hyperferritinemic syndrome, an immunomodulatory perspective to alleviate the storm. Front Immunol 2020;11:1130.

[23] Shaeib F, Khan S N, Ali I, Najafi T, Maitra D, Abdulhamid I, Saed G M, Pennathur S, Abu-Soud H M. Melatonin prevents myeloperoxidase heme destruction and the generation of free iron mediated by self-generated hypochlorous acid PLoS ONE. 2015; 10(4): e0120737.

[24] Habib HM, Ibrahim S, Zaim A, Ibrahim WH. The role of iron in the pathogenesis of COVID-19 and possible treatment with lactoferrin and other iron chelators. Biomed Pharmacother 2021;136:111228. https://doi.org/10.1016/j.biopha.2021.111228.

[25] Collin F. Chemical basis of reactive oxygen species reactivity and involvement in neurodegenerative diseases Int. J. Mol. Sci. 2019;20(10):2407. https://doi. org/10.3390/ijms20102407.

[26] Li J, Cao F, Yin H-liang, Huang Z-jian, Lin Z-tao, Mao N, et al. Ferroptosis: past, present and future. Cell Death Dis 2020;11(2). https://doi.org/10.1038/s41419020-2298-2.

[27] Yang M, Lai CL. SARS-CoV-2 infection: can ferroptosis be a potential treatment target for multiple organ involvement? Cell Death Discovery 2020;6:130.

[28] Slominski AT, Hardeland R, Zmijewski MA, Slominski RM, Reiter RJ, Paus R. Melatonin: a cutaneous perspective on its production, metabolism, and functions. J, Invest Dermatol 2018;138(3):490-9.

[29] Gulcin I, Buyukokuroglu ME, Kufrevioglu OI. Metal chelating and hydrogen peroxide scavenging effects of melatonin. J Pineal Res. 2003;34(4):278-81.

[30] Abd Elkader MAE, Aly HH. Protective effect of melatonin against iron overloadinduced toxicity in rats. Int J Pharm Pharm Sci 2015;7(9):116-21.

[31] Faramarzi M, Sadighi M, Chitsazi M, Esmailzadeh M, Foladvand G. The effect of adjunctive use of melatonin as a supplement on serum ferritin level in periodontal patients: a randomized, controlled trial. Dental Research Journal 2021;18(1):39.

[32] LABONIA WALTER, RUBIO DEBORA, ARIAS CARLOS. Melatonin corrects reticuloendothelial blockade and iron status in haemodialysed patients. Nephrology (Carlton). 2005;10(6):583-7.

[33] Favero G, Franceschetti L, Bonomini F, Rodella LF, Rezzani R. Melatonin as an antiinflammatory agent modulating inflammasome activation. International Journal of Endocrinology. 2017;2017:1-13.

[34] Ferreira AndréC, Soares VC, de Azevedo-Quintanilha IG, Dias SdaSG, FintelmanRodrigues N, Sacramento CQ, et al. SARS-CoV-2 engages inflammasome and pyroptosis in human primary monocytes. Cell Death Discovery 2021;7(1). https:// doi.org/10.1038/s41420-021-00428-w.

[35] Gou Z, Su X, Hu X, Zhou Y, Huang L, Fan Y, et al. Melatonin improves hypoxic ischemic brain damage through the Akt/Nrf2/Gpx4 signaling pathway. Brain Res Bull 2020;163:40-8.

[36] Baltaci A, Mogulkoc R. Pinealectomy and melatonin administration in rats: their effects on plasma leptin levels and relationship with zinc. Acta Biol Hung. 2007;58 (4):335-43.

[37] Jothimani D, Kailasam E, Danielraj S, Nallathambi B, Ramachandran H, Sekar P, et al. COVID-19: Poor outcomes in patients with zinc deficiency. Int J Infect Dis. 2020;100:343-9.

[38] Park H-S, Lee SC, Cardenas ME, Heitman J. Calcium-calmodulin-calcineurin signaling: a globally conserved virulence cascade in eukaryotic microbial pathogens. Cell Host Microbe. 2019;26(4):453-62.

[39] Benítez-King G, Ríos A, Martínez A, Antón-Tay F. In vitro inhibition of $\mathrm{Ca}^{2+}$ / calmodulin-dependent kinase II activity by melatonin. Biochim. Biophys. Acta. 1996;1290:191-6.

[40] Artis WM, Fountain JA, Delcher HK, Jones HE. A mechanism of susceptibility to mucormycosis in diabetic ketoacidosis: transferrin and iron availability. Diabetes 1982;31(12):1109-14.

[41] Aouichat S, Navarro-Alarcon M, Alarcón-Guijo P, Salagre D, Ncir M, Zourgui L et al. Melatonin improves endoplasmic reticulum stress-mediated IRE1 $\alpha$ pathway in Zücker diabetic fatty rat. Pharmaceuticals (Basel). 2021;14(3):232. https://doi. org/10.3390/ph14030232.

[42] El-Missiry MA, El-Missiry ZMA, Othman AI. Melatonin is a potential adjuvant to improve clinical outcomes in individuals with obesity and diabetes with coexistence of Covid-19. Eur J Pharmacol 2020;882:173329. https://doi.org/ 10.1016/j.ejphar.2020.173329.

[43] Gupta S, Haldar C. Physiological crosstalk between melatonin and glucocorticoid receptor modulates T-cell mediated immune responses in a wild tropical rodent, Funambulus pennanti. J Steroid Biochem Mol Biol. 2013;134:23-36.

[44] Castellano-Gonzalez G, Clancy LE, Gottlieb D. Prospects for adoptive T-cell therapy for invasive fungal disease. Curr Opin Infect Dis. 2017;30(6):518-27.

[45] Savage R A, Zafar N, Yohannan S, Miller J-M M. Melatonin. StatPearls NCBI Bookshelf. 2021; StatPearls Pub., https://www.ncbi.nlm.nih.gov/books/ NBK534823/. 
[46] Zhou Y, Hou Y, Shen J, Kallianpur A, Zein J, Culver D A et al. A network medicine approach to investigation and population-based validation of disease

manifestations and drug repurposing for COVID-19. PLoS Biol 2020;18: e3000970.

[47] Tesarik J. Melatonin attenuates growth factor receptor signaling required for SARSCoV-2 replication. Melatonin Res. 2020;3(4):534-7.
[48] Reynolds JL, Dubocovich ML. Melatonin multifaceted pharmacological actions on melatonin receptors converging to abrogate COVID-19. J Pineal Res. 2021;71(1) https://doi.org/10.1111/jpi.v71.110.1111/jpi.12732. 